\title{
Density Fluctuations in Glassy Poly(ethylene terephthalate). Influence of the Thermal Treatment
}

\author{
Mari Cruz García, Daniel Reyes Rueda, and Francisco Jose Baltá Calleja
}

Instituto de Estructura de la Materia, CSIC, Serrano 119, 28006 Madrid, Spain

(Received January 14, 1999)

KEY WORDS Electron Density Fluctuations / Simultaneous Small-Angle X-Ray Scattering and Wide-Angle X-Ray Scattering / Cold Crystallization / Poly(ethylene terephthalate) /

In preceding studies it has been reported that, during isothermal crystallization of glassy poly(ethylene terephthalate) (PET) and polyaryl(ether ketone ketone) (PEKK) samples, the small-angle X-ray scattering (SAXS) maximum develops before the appearance of the wide-angle (WAXS) crystalline peaks. ${ }^{1,2}$ In these studies the SAXS peak was originated by long range density fluctuations of the order of $8-20 \mathrm{~nm}$. On the other hand, WAXS crystalline peaks were brought about by the 3-dimensional order within the crystalline lamellae. The appearance of the SAXS maxima before the WAXS peaks suggests that long range density fluctuations are a precursor during the induction period prior to primary crystallization. Chain parallelization prior to crystallization in polymers is a process whose kinetics has been described in terms of the spinodal decomposition formalism. ${ }^{3,4}$ According to this concept, during the early stages of phase separation the density fluctuations increase exponentially with time while the wave vector of the fluctuation mode remains constant. The phase separation can be analyzed in this case in terms of the Cahn-Hilliard-Cook's theory. ${ }^{5}$

The aim of the present study is to contribute to the understanding and clarification of the embryonic chain ordering mechanism prior to crystallization. The time scale for electron density fluctuations during cold crystallization of PET measured by real time X-ray scattering experiments has been shown to depend on the temperature of crystallization. ${ }^{6}$ In the present investigation we wish to examine the dependence of the time scale for electron density fluctuations upon the nature of the starting glassy state.

$\mathrm{X}$-ray scattering experiments using synchrotron radiation were performed aiming to examine the influence of the nature of glassy state, previously induced by heat treatment, on the cold crystallization features from the glass. Amorphous PET thin films, ( $\sim 200 \mu \mathrm{m}$ thick), without nucleating agent supplied by Kalle, A. G., Germany were used in this study (sample a). The transparent original PET films had a glass transition temperature of $\sim 75^{\circ} \mathrm{C}$. Two further amorphous samples were prepared by annealing of the original films at $T=95^{\circ} \mathrm{C}$ for times $\left(t_{\mathrm{a}}\right)$ of $1.5 \mathrm{~h}$ (sample b) and $5 \mathrm{~h}$ (sample c), respectively. The three starting samples are glassy, and show a similar amorphous halo (see Figure 1).

It is known that PET without nucleating agents, annealed at a given temperature, takes much longer times to crystallize than PET with nucleating agents. ${ }^{7,8}$
Thus, the fact that in ref 1 it was reported that PET film starts to crystallize at $85^{\circ} \mathrm{C}$ after an induction period of $120 \mathrm{~min}$, in contrast with the above sample c (which remains amorphous after annealing at $95^{\circ} \mathrm{C}$ for $5 \mathrm{~h}$ ) might be explained in terms of the different additives contained in each sample.

Simultaneous WAXS and SAXS experiments were carried out using a double-focussing mirror monochromator camera on the polymer beam line (A2) at HASYLAB (Hamburg, FRG). Cold crystallization was performed isothermally bringing up each glassy sample instantly $\left(\sim 100^{\circ} \mathrm{C} \mathrm{min}^{-1}\right)$ to the crystallization temperature $\left(T_{\mathrm{c}}=105^{\circ} \mathrm{C}\right)$. Scattering patterns were recorded by using linear position sensitive detectors and corrected for fluctuations in intensity of the primary beam and background. The data acquisition system is based on CAMAC hardware and modulation software. ${ }^{9}$ The measurement of the SAXS invariant was carried out in the $s$ range $0.01 \mathrm{~nm}^{-1}<s<0.17 \mathrm{~nm}^{-1}(s=2 \sin \theta / \lambda$ and $\lambda=0.150 \mathrm{~nm}$ ).

Figure 2 illustrates, as an example, the time variation of WAXS and SAXS patterns of sample c, with reference to the first frame recorded at $T_{\mathrm{c}}=105^{\circ} \mathrm{C}$, during isothermal crystallization.

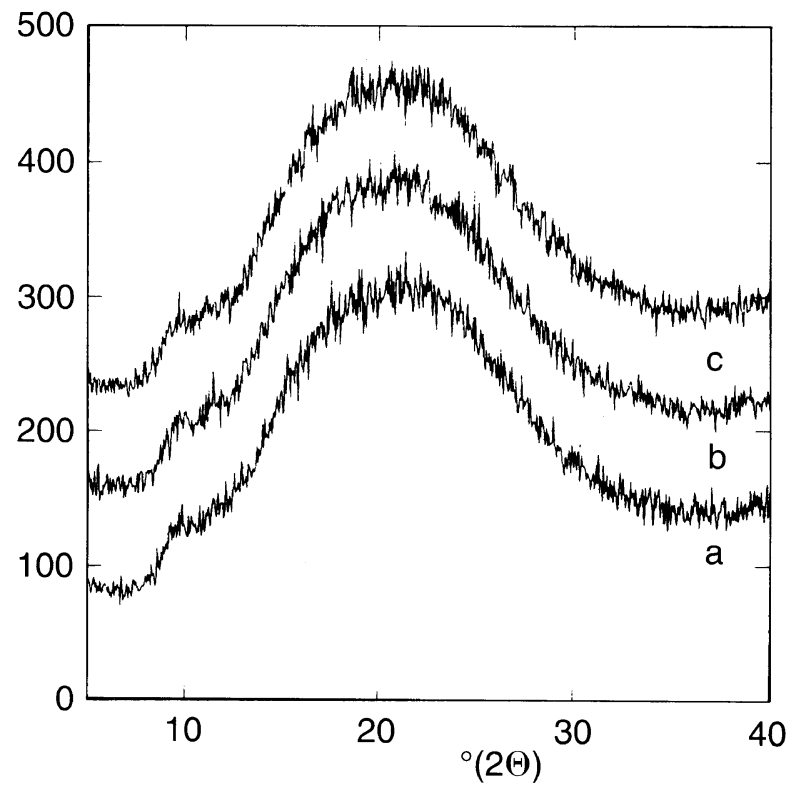

Figure 1. WAXS diffractograms, recorded at room temperature, of amorphous PET before (sample a) and after heat treatment at $95^{\circ} \mathrm{C}$ for: (b) $1.5 \mathrm{~h}$ and (c) $5 \mathrm{~h}$. 

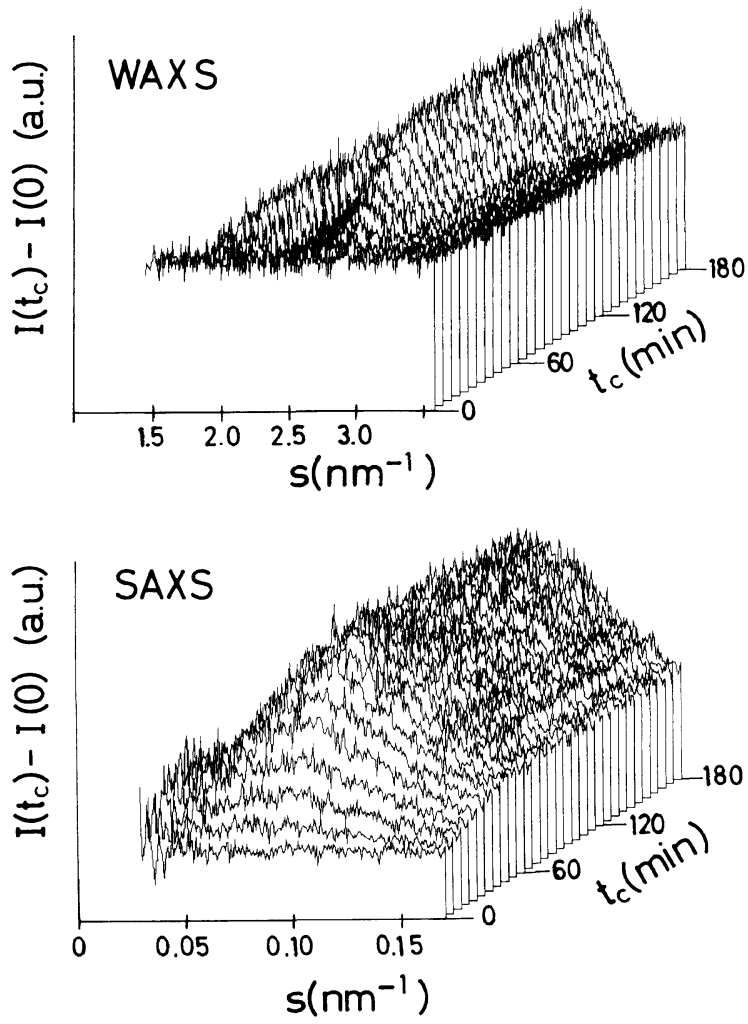

Figure 2. Variation of wide (top) and small-angle X-ray scattering (bottom) during cold isothermal crystallization of PET (sample b) at $T_{\mathrm{c}}=105^{\circ} \mathrm{C}$ (with reference to the first frame at $T_{\mathrm{c}}=105^{\circ} \mathrm{C}$ ).

Figure 3 shows the variation of the invariant, $Q$ and the X-ray crystallinity, $w_{\mathrm{c}}$ as a function of time for an isothermal experiment carried out at $T_{\mathrm{c}}=105^{\circ} \mathrm{C}$ for the three amorphous PET samples investigated. The data of Figure 3 clearly show that the SAXS intensity maximum grows before the appearance of the WAXS crystallinity for each sample as already reported in previous studies. ${ }^{1,2}$

The time evolution of the integrated SAXS intensity $I\left(t_{\mathrm{c}}\right)$ (referred to the first frame) at $105^{\circ} \mathrm{C}$ in the above range is shown, for the original PET film (sample a), in Figure 4. From the early times of the induction period a gradual increase of $I\left(t_{\mathrm{c}}\right)$ for the isothermal process is observed. For $t_{\mathrm{c}}>27 \mathrm{~min}$ at $105^{\circ} \mathrm{C}$ the onset of crystallization is characterized by a greater rate of increase of $I\left(t_{\mathrm{c}}\right)$ which is concurrent with the appearance of Bragg reflections and an increase in the total area of the WAXS peaks. During the early times of the isothermal process $I\left(t_{\mathrm{c}}\right)$ increases exponentially with time as shown in the insert of Figure 4. This time dependence agrees well with the CHS's linearized theory for early stages of spinodal decomposition. ${ }^{5}$

Most interesting is, however, the fact that the precrystallization induction period depends on the annealing time of the amorphous PET samples; longer annealing times leading to shorter induction periods (Figure 3). This finding suggests that adequate annealing of the glass before any crystallization occurs, may contribute to a local rearrangement of polymer chains 'frozen-in' in denser regions, showing density fluctuations, without crystal nuclei formation. Figure 5 illustrates the SAXS intensity, recorded at room temperature, for the amorphous samples $\mathbf{c}\left(t_{\mathrm{a}}=5 \mathrm{~h}\right.$ at $\left.T=95^{\circ} \mathrm{C}\right)$

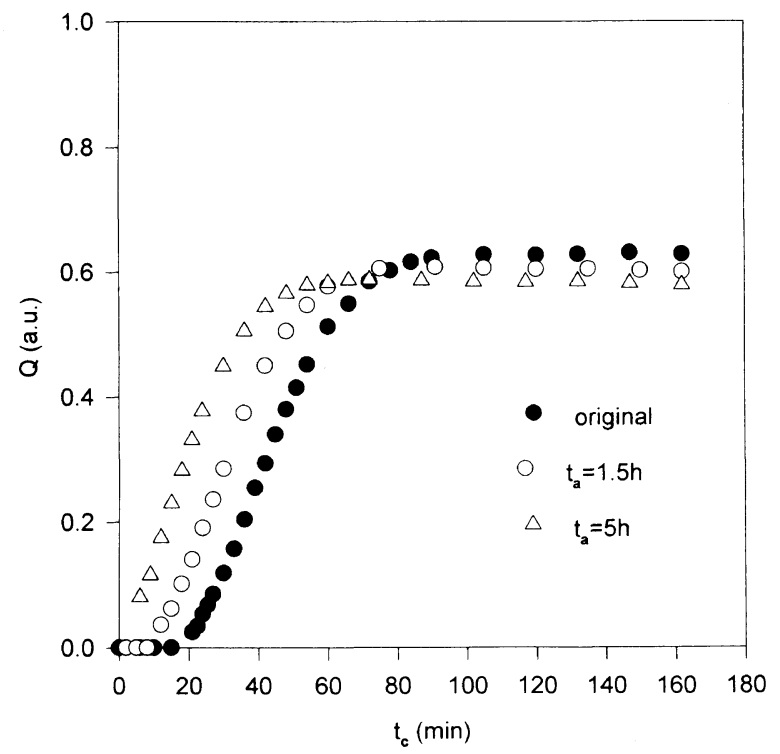

(a)

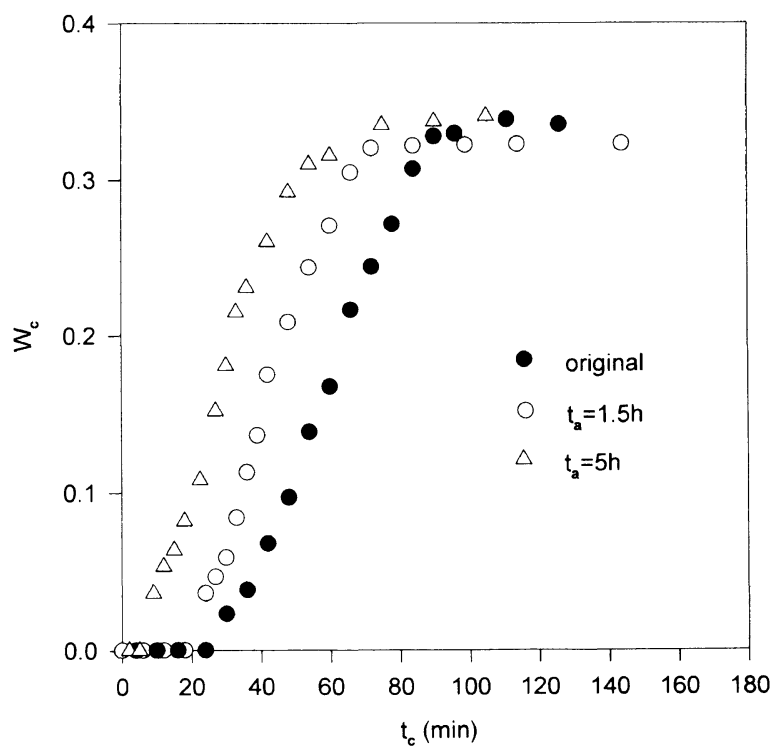

(b)

Figure 3. (a) SAXS invariant, $Q$, and (b) WAXS index of crystallinity $w_{\mathrm{c}}$, of the samples of Figure 1 during isothermal crystallization $\left(T_{\mathrm{c}}=105^{\circ} \mathrm{C}\right)$, vs. crystallization time, $t_{\mathrm{c}}$.

and a (original sample). Though, at first sight, one observes a continuous character for both intensity profiles, the intensity difference between both profiles (samples $\mathbf{c}$ and a) shows the appearance of an excess of intensity in the $s$-range investigated (insert). This intensity difference could be associated to the 'frozen-in' electron density fluctuation arising from the sample c prior to crystallization.

The evolution of the SAXS patterns with time exhibits the appearance of a maximum with a periodicity $L$ which gradually decreases with crystallization time (Figure 6). For short heating times, $L$ corresponds to the presence of density fluctuations in the sample. For longer times $L$ corresponds to the stacking of incipient lamellae.

For sample $\mathbf{c}$ the long range density fluctuations give rise to a maximum at about $L=9.5 \mathrm{~nm}$ after an annealing time of $t_{\mathrm{c}} \sim 0.5 \mathrm{~min}$ before crystallization occurs. 


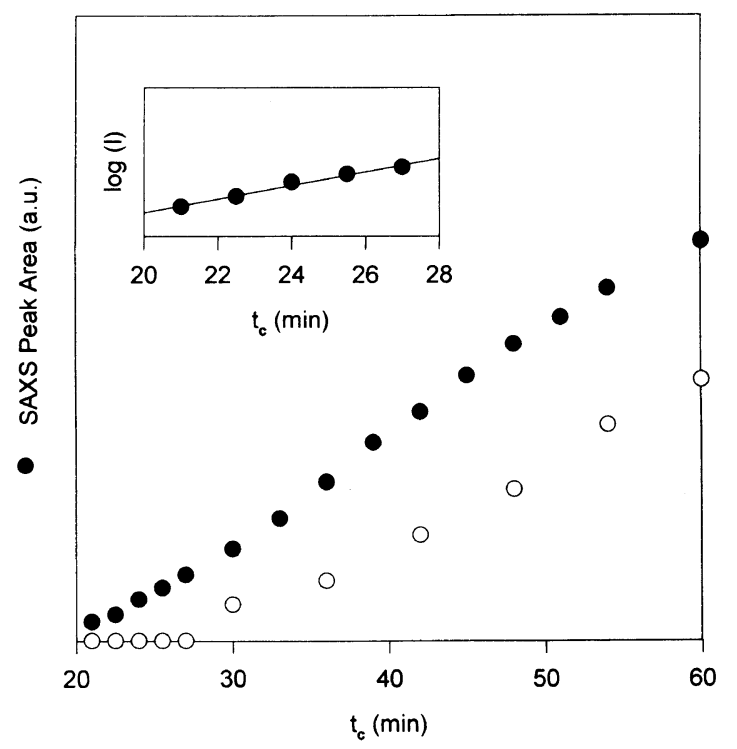

Figure 4. SAXS and WAXS integrated intensity for amorphous PET (sample a) isothermally crystallized $\left(T_{\mathrm{c}}=105^{\circ} \mathrm{C}\right) \mathrm{vs}$. $t_{\mathrm{c}}$. Insert: plot of $\log I_{\text {SAXS }} v$ s. $t_{\mathrm{c}}$

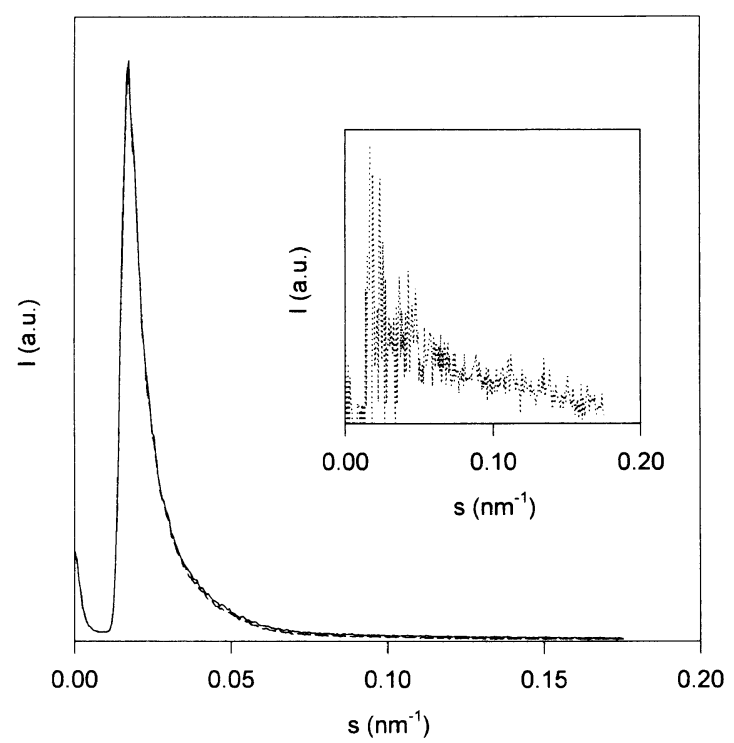

Figure 5. SAXS profiles for samples a and $\mathbf{c}$. Insert: SAXS intensity difference between profiles of samples $\mathbf{c}$ and $\mathbf{a}$.

Similarly, density fluctuations, during the induction period of samples $\mathbf{b}$ and $\mathbf{a}$ lead to scattering maxima at about $10.0 \mathrm{~nm}$ and $10.3 \mathrm{~nm}$ respectively. It is noteworthy that the fluctuation range (L-value) depends on the initial annealing time $\left(\right.$ at $95^{\circ} \mathrm{C}$ ) of the sample, the $L$-values becoming shorter the larger $t_{\mathrm{a}}$.

The $L$ values obtained (Figure 6) fit well with the thicknesses of lamellae $(7.5 \mathrm{~nm})$ measured from electron micrographs during the first stages of crystallization. ${ }^{10}$ For longer $t_{\mathrm{c}}$ annealing times $\left(t_{\mathrm{c}}>30 \mathrm{~min}\right.$ for sample a) $t_{\mathrm{c}}>25 \mathrm{~min}$ for sample $\mathbf{b} ; t_{\mathrm{c}}>15 \mathrm{~min}$ for sample $\left.\mathbf{c}\right)$ crystallinity gradually develops (see Figure $3 b$ ) and the SAXS maxima correspond to the lamellae periodicity.

According to the dual lamellar stack model, the average long period decrease shown in Figure 6 for $t_{\mathrm{c}}$ $>30$ min could be consistent with the formation of secondary lamellar stacks which have a smaller long

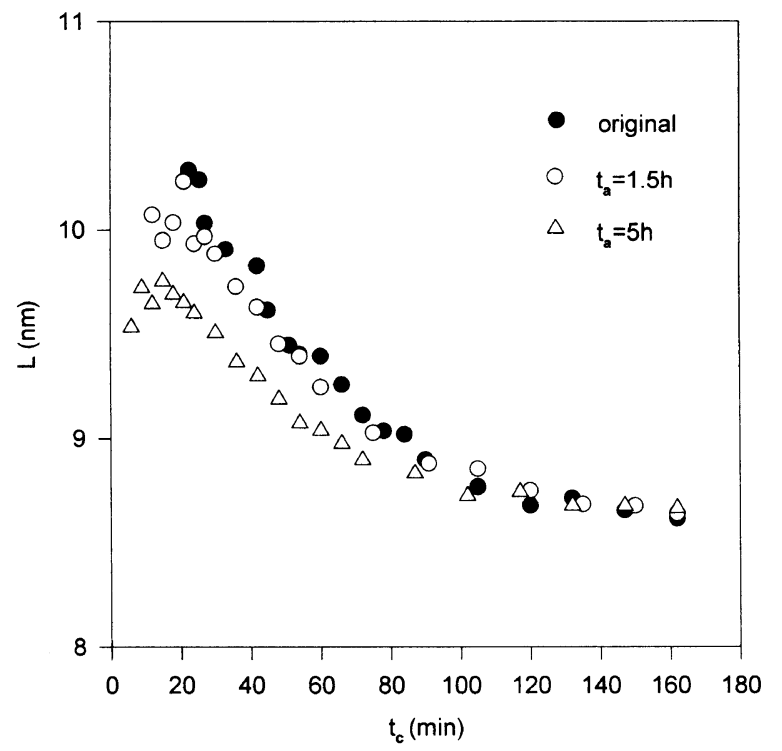

Figure 6. Dependence of long period $L$ for isothermally treated $\left(T_{\mathrm{c}}=105^{\circ} \mathrm{C}\right)$ glassy PET samples as a function of $t_{\mathrm{c}}$.

period as compared to the primary lamellar stacks. ${ }^{11,12}$ On the other hand, according to the lamellar insertion model, the long period decrease could also be due to the insertion of thinner lamellae between the pre-existing ones. ${ }^{13,14}$

In conclusion, the present results suggest that the time scale for electron density fluctuations prior to cold crystallization of PET strongly depends on the local arrangement of polymer chains within the glassy state induced by the different heat treatments of the starting material.

Acknowledgments. Grateful acknowledgment is due to the International Joint Research Grant NEDO and to the DGICYT, Spain (Grant PB94-0049) for the support of this investigation.

\section{REFERENCES}

1. M. Imai, K. Mori, T. Mizukami, K. Kaji, and T. Kanaya, Polymer, 33, 4451 (1992).

2. T. A. Ezquerra, E. López Cabarcos, B. S. Hsiao, and F. J. Baltá Calleja, Phys. Rev. E., 54, 989 (1996).

3. K. Binder, "Materials Science and Technlogy," Vol. 5, Phase Transition, V.C.H. Publ., Weinheim, 1993.

4. G. Strobl, "The Physics of Polymers," Springer, Berlin, 1996, p 173.

5. J. W. Cahn and J. H. Hilliard, J. Chem. Phys., 28, 258 (1958).

6. M. C. García, D. R. Rueda, and F. J. Baltá Calleja, "HASYLAB, Hamburg, Annual Report," 1997, p 717.

7. T. Asano, A. Dzeick-Pickuth, and H. G. Zachmann, J. Mater. Sci., 24, 1967 (1989).

8. F. J. Baltá Calleja, C. Santa Cruz, and T. Asano, J. Polym. Sci., Polym. Phys., 31, 557 (1993).

9. C. Bonhn, R. Kempf., M. H. H. Koch, and S. M. McLaughin, Nucl. Instr. Methods Phys. Res. Sect., A 249, 399 (1986).

10. F. J. Baltá Calleja, D. R. Rueda, G. H. Michler, and I. Naumann, J. Macromol. Sci.-Phys., B37, 411 (1998).

11. M. P. Lathmer, J. K. Hobbs, M. J. Hill, and P. J. Barham, Polymer, 33, 3971 (1992).

12. R. K. Verma and B. S. Hsiao, Trends Polym. Sci., 4, 352 (1996).

13. B. S. Hsiao, K. H. Gardner, D. Q. Wu, and B. Chu, Polymer., 34, 3986 (1993).

14. K. N. Krüger and H. G. Zachmann, Macromolecules, 26, 5202 (1993). 